\title{
Personality Attributes of Secondary Nocturnal Enuresis Using Exner's Rorschach Comprehensive System
}

\author{
Dr.Asmaa Othman Diab Abd-Elmaksoud \\ Lecturer at Department of Mental Health, \\ Faculty of Education in the New Valley, \\ Assiut University
}

\begin{abstract}
:
This study investigated Rorschach Inkblot Test response patterns in a group of children diagnosed with secondary nocturnal enuresis and compared them to normal group. The mean age was 13.9. A total of 7 children out of 550 had secondary nocturnal enuresis. Four children completed the Rorschach Inkblot test. Four children without secondary nocturnal enuresis were included in the control group. Responses scored using Exner's Comprehesive system. The findings indicated that enuretics differ significantly from the normals on affect, self perception and interpersonal perception. Successful programs should focus on enhancing self-esteem and reduce anxiety and depression in the enuretic child.
\end{abstract}

Keywords: Personality Attributes, Nocturnal Enuresis, Rorschach Inkblot Test. 


\section{בersonality Attributes of Secondary Nocturnal Enuresis}

\section{Introduction:}

According to International Children's Continence Society (ICCS), nocturnal enuresis (NE) is defined as intermittent incontinence while asleep in children older than 5 years age in which the sphincters control is acquired (Nevéus, von Gontard, Hoebeke, et al, 2006). Enuresis is estimated to affect 5 to 7 million US children $\geq$ 6 years of age (National Kidney Foundation Home Page. Available, 2003). Yearly, the spontaneous rate of resolution for nocturnal enuresis is $\sim 15 \%$ (Theunis, Hoecke, Paesbrugge, Hoebeke, Walle, 2002).

Nocturnal enuresis can be present with or without lower urinary tract (LUT) symptoms. When only NE is present, the disorder is referred to as monosymptomatic enuresis. In the presence of other symptoms, the disorder is referred to as nonmonosymptomatic enuresis (Austin, Bauer, Bower, Chase, Franco, 2014).

Nocturnal enuresis is subdivided into primary and secondary forms. Primary enuresis is the presence of enuresis in a child $\geq 5$ years old who has never achieved an asymptomatic period ( $\geq 6$ months) of consistent nighttime dryness. Secondary enuresis is the presence of enuresis in a child $\geq 5$ years old who has achieved an asymptomatic period ( $\geq 6$ months) of consistent nighttime dryness in the past (Von Gontard, Mauer-Mucke, Plück, Berner, Lehmkuhl, 1999). Secondary enuresis is most commonly triggered by an unusually stressful life event, significant enough to cause psychosocial regression (Robson and Leung, 2000).

The prevalence of NE has been studied in multiple regions and countries, and values have varied according to what is thought to be a difference in attitude and expectations towards this problem. The NE prevalence was reported as following: $18.6 \%$ in India, $3.8 \%$ in Italy, 9.2\% in Korea, $8 \%$ in Malaysia, $5.5 \%$ in Taiwan, $4.2 \%$ in Thailand, $12.4 \%$ in Turkey, $18.9 \%$ in Australia, $15 \%$ in Saudi Arabia, $3.5 \%$ in Hong Kong, $6 \%$ in The Netherlands, $7.4 \%$ in New Zealand, $13.7 \%$ in Sudan, and $6.8 \%$ in Iran (Safarinejad, 2007). The prevalence rate of nocturnal enuresis in Egypt among children of 5-12 years old joining a primary governate school in Assiut city was 17.8\% (Hammad, El-

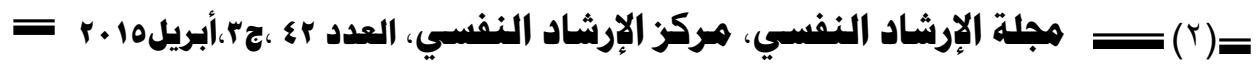




\section{Dr.Asmaa Othman Diab}

Sedfy, Ahmed, 2005). and in Benha city was 15.7\% (Mohammed, Saleh, Al Zoheiry, 2014).

The wide prevalence of NE speaks to the need for an improved understanding of this condition by patients, families, pediatricians, primary care doctors and urologists alike. The association of NE with many other conditions, such as: Cognitive problems, low self esteem, Coppola, Costantini, Gaita, \& Saraulli, 2011), attention deficit hyperactivity disorder and disturbed sleep (Mellon, Natchev, Katusic, Colligan, et al., 2013), enlarges the need for all clinicians to be able to recognize and evaluate patients with this disorder

Despite the significant problems that NE children experience, little effort has been made to understand the psychodynamics of enuretics using standardized test instruments, such as the Rorschach Inkblot Test. This study attempts to focus on and examine the underlying personality attributes of children diagnosed as secondary $\mathrm{NE}$ as demonstrated on the Rorschach Inkblot Test, a procedure frequently used as part of an overall psychological evaluation.

\section{Materials and Methods:}

\section{Study Subjects:}

This study was conducted in preparatory school children. They were selected from Al-Kharga city in the New Valley governorate. The sample size was 550 (females 360 and 190 males).

This was a two-step study, the first step was a preliminary brief questionnaire distributed to the students within sealed envelopes in order to avoid embarrassment of the children, and they were asked to take it home to their parents. This questionnaire asked about the presence of enuresis, and if present, whether it occurs during the day only, at night only, or day and night. These questionnaires were analyzed and only the individuals with enuresis at night were included in the second part of the study.

The second step included individually meeting with every child who met the inclusion criteria and his/her parents. Exclusion criteria were, urogenital malformations, renal failure, chronic disease such as diabetes and students who had never achieved an asymptomatic period ( $\geq 6$ months) of consistent nighttime dryness.

7 patients were diagnosed to have nocturnal enuresis in this study with a prevalence rate of $1.3 \%$. All patients were expected to

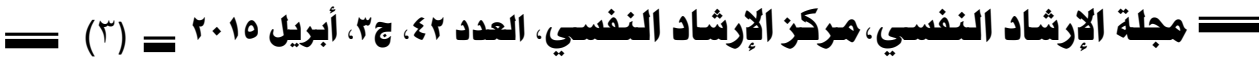


complete the Rorschach and then scored with the Exner Comprehensive System (CS) (Exner, 1974, 1978). Three patients did not complete the Rorschach (2boys and one girl). A normal control group $(n=4)$ was selected from the same sample who proved not to have enuresis at all. Children in the two groups were matched by age (mean age 13 years and 9 months) and gender (females) and IQs obtained for the two groups using Stanford Binet Intelligence scale, fourth Edition with a mean IQ $92(\mathrm{SD}=3.4)$.

\section{Results:}

Table 1 displays the mean, sum of ranks, and group differences on the RIM variables on the number of responses.

Table (1)

Differences between enuretics $(n=4)$ and a comparison group $(n=4)$

on the number of responses.

\begin{tabular}{|c|c|c|c|c|c|}
\hline Variable & $\begin{array}{l}\text { Comparison } \\
\text { groups }\end{array}$ & $\begin{array}{l}\text { Mean of } \\
\text { ranks }\end{array}$ & $\begin{array}{l}\text { Sum of } \\
\text { ranks }\end{array}$ & $\mathbf{U}^{\text {a value }}$ & Sig. \\
\hline $\begin{array}{l}\text { Number of } \\
\text { Responses }\end{array}$ & enuretics & 4.25 & 17 & \multirow{2}{*}{0.296} & ns \\
\cline { 2 - 4 } & normals & 4.75 & 19 & \\
\hline
\end{tabular}

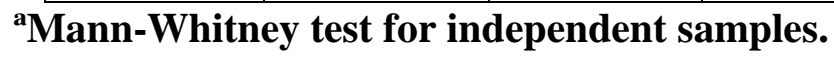

It is important to note that the two groups did not differ in terms of the number of responses (R) given, and therefore group differences cannot be simply attributed to overall productivity. The Rorschach response reflects the withdrawal, inwardization, and restriction that the 13-year-old shows clearly in other life situations. The number of responses given to the Rorschach cards increased steadily between 10 and 12 years. At 13, it declines to a median of 17 .

Table (2) displays the mean, sum of ranks, and group differences on the RIM variables on Lambda.

Table (2)

Differences between enuretics $(n=4)$ and a comparison group $(n=4)$ on

Lambda

\begin{tabular}{|c|l|l|l|l|l|}
\hline Variable & $\begin{array}{l}\text { Comparison } \\
\text { groups }\end{array}$ & $\begin{array}{l}\text { Mean of } \\
\text { ranks }\end{array}$ & $\begin{array}{l}\text { Sum of } \\
\text { ranks }\end{array}$ & $\mathbf{U}^{\text {a }}$ value & Sig. \\
\hline Lambda & enuretics & $\mathbf{5 . 2 5}$ & 21 & \multirow{2}{*}{0.871} & ns \\
\cline { 2 - 5 } & normals & 3.75 & 15 & 0.871 \\
\hline
\end{tabular}

Lambda is a key variable in the Rorschach Comprehensive System (CS). It is calculated as the ratio of pure form responses to

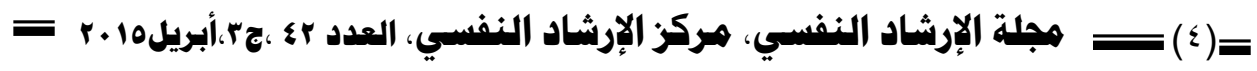


nonpure form responses. Specifically, Lambda $=F /(R-F)$, where $F$ indicates the number of pure form responses, and $\mathrm{R}$ indicates the total number of responses.

In terms of percentages and ratios, there were no significant differences between the groups in terms of percentage of pure form responses (Lambda).

Table (3) displays the mean, sum of ranks, and group differences on Affect

Table (3)

Differences between enuretics $(n=4)$ and a comparison group $(n=4)$ on Affect

\begin{tabular}{|c|c|c|c|c|c|}
\hline $\begin{array}{l}\text { Variable } \\
\text { (Affect) }\end{array}$ & $\begin{array}{l}\text { Comparison } \\
\text { groups }\end{array}$ & $\begin{array}{ll}\text { Mean of } \\
\text { ranks }\end{array}$ & $\begin{array}{l}\text { Sum of } \\
\text { ranks }\end{array}$ & $\begin{array}{l}\mathbf{U}^{\mathbf{a}} \\
\text { value }\end{array}$ & Sig. \\
\hline \multirow[t]{2}{*}{ Afr } & enuretics & 2.88 & 11.50 & \multirow{2}{*}{1.888} & \multirow{2}{*}{ ns } \\
\hline & normals & 6.12 & 24.50 & & \\
\hline \multirow[t]{2}{*}{ Blends } & enuretics & 2.50 & 10 & \multirow{2}{*}{2.309} & \multirow{2}{*}{0.05} \\
\hline & normals & 6.50 & 26 & & \\
\hline \multirow[t]{2}{*}{ Sum C } & enuretics & 3 & 12 & \multirow{2}{*}{1.845} & \multirow{2}{*}{ ns } \\
\hline & normals & 6 & 24 & & \\
\hline \multirow[t]{2}{*}{ Sum $C^{\prime}$} & enuretics & 6.50 & 26 & \multirow{2}{*}{2.352} & \multirow[t]{2}{*}{0.05} \\
\hline & normals & 2.50 & 10 & & \\
\hline
\end{tabular}

The Affect cluster revealed significant effects by group in Blends and Sum $C^{\prime}$. The two groups did not differ in terms of Affective Ratio (Afr) This ratio is comprised of the total number of responses to the last three cards, compared with those given to the first seven cards. Sum R(VIII + IX $+\mathrm{X}) / \mathrm{Sum} \mathrm{R}(\mathrm{I}-\mathrm{VIII})$. This variable relates to one's interest in experiencing or being around emotional stimuli. The enuretics produced fewer Sum C' responses and fewer Blends than the normal group. These data indicate that the marked tendency to narrow and simplify the stimulus fields presented, results in an overcontrol of impulse expression through withdrawal from emotion-arousing stimuli and in negative affects and feelings related to internal distress and discomfort. The $\mathrm{C}^{\prime}$ responses in enuretics represent some form of conscious control or defense against affective expression. In a broad sense, it is an anxious or cautions form of adaptation wherein the cognitive elements thwart affective expression. The $C^{\prime}$ responses also represent some index of depressive and helplessness feelings as the excessive use of $\mathrm{C}^{\prime}$ as "black" is thought to

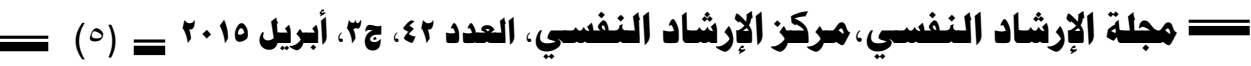


reflect feelings of rejection often expressive of true depression. The study found no statistical differences between the two groups in Sum $\mathrm{C}$ responses. This can be explained by the hypotheses that median $\Sigma \mathrm{C}$ is lower in the age of thirteen than at any other age (Ames, Metraux, and Walker, 1959).

Table (4) displays the mean, sum of ranks, and group differences on Self-perception

Table (4)

Differences between enuretics $(n=4)$ and a comparison group $(n=4)$ on Self-

perception

\begin{tabular}{|l|l|l|l|l|l|}
\hline Variable & $\begin{array}{l}\text { Comparison } \\
\text { groups }\end{array}$ & $\begin{array}{l}\text { Mean of } \\
\text { ranks }\end{array}$ & $\begin{array}{l}\text { Sum of } \\
\text { ranks }\end{array}$ & $\begin{array}{l}\mathbf{U}^{\text {a }} \\
\text { value }\end{array}$ & Sig. \\
\hline $\begin{array}{l}\text { Self- } \\
\text { perception } \\
3 \mathrm{r}+(2) / \mathrm{R}\end{array}$ & enuretics & 2.50 & 10 & & \multirow{2}{*}{2.323} \\
\cline { 2 - 5 } & normals & 6.50 & 26 & & \\
\hline
\end{tabular}

The Exner (1974, 1978) Comprehensive Rorschach system produces an Ego-centricity Index defined as $3 r+(2) / R$, where $r=$ the number of reflection responses, $(2)=$ the number of pair answers, and $\mathrm{R}=$ the total number of responses in the protocol. This ratio provides an index of self concern, self-focusing or egocentricity. Early findings suggested that an excessively high or low index may accompany psychopathological states.

On the Self Perception cluster, the enuretics produced significantly lower Egocentricity Index scores when compared to the normal group. These data indicate that the enuretics experience generally lower self-esteem and tend to make more negative judgments about the relationship between self and others.

Table (5) displays the mean, sum of ranks, and group differences on Interpersonal perception

$$
\text { Table (5) }
$$

Differences between enuretics $(n=4)$ and a comparison group $(n=4)$ on Interpersonal

\begin{tabular}{|l|l|l|l|l|l|}
\hline $\begin{array}{c}\text { Variable } \\
\begin{array}{c}\text { Interpersonal } \\
\text { perception }\end{array}\end{array}$ & $\begin{array}{c}\text { Comparison } \\
\text { groups }\end{array}$ & $\begin{array}{c}\text { Mean of } \\
\text { ranks }\end{array}$ & $\begin{array}{c}\text { Sum of } \\
\text { ranks }\end{array}$ & $\mathbf{U}^{\text {a value }}$ & Sig. \\
\hline Cop & enuretics & 2.50 & 10 & 2.397 & 0.05 \\
\cline { 2 - 6 } & normals & 6.50 & 26 & 2.337 & 0.05 \\
\hline Pure H & enuretics & 2.50 & 10 & 26 & \\
\cline { 2 - 5 } & normals & 6.50 & 26 & & \\
\hline
\end{tabular}

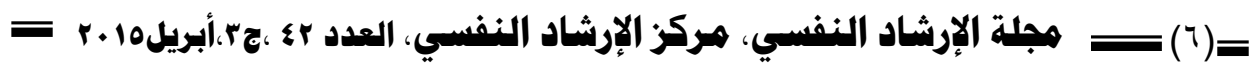




\section{-Dr.Asmaa Othman Diab}

On the Interpersonal Perception cluster, the enuretics produced significantly fewer COP and fewer Pure $\mathrm{H}$ than the comparison group. These data suggest that enuretics experience less interest or willingness to engage others actively and that they feel less secure and comfortable in interpersonal situations.

\section{Discussion}

The results of this study indicate that neurotics differ significantly from the normals on a number of Rorschach variables. The study found no statistical differences between the two groups in terms of the number of responses (R) given, and in terms of percentage of pure form responses (Lambda), therefore group differences cannot be simply attributed to overall productivity. These findings are consistent with the study of Yu, Kong, Peng, Ma, Liu, Guo (2012) which found that full intelligence quotient (FIQ), verbal IQ (VIQ), performance IQ (PIQ), were within the normal range and did not significantly differ from the control group.

The study also found that neurotics suffer from withdrawal, anxiety, depression and low self-esteem as it appears from the affect cluster especially from achromatic color $\left(C^{\prime}\right)$ which relate to irritating feelings that are caused by the inhibition or internalization of emotion. All people do this now and then in various situations, but some people do it more frequently and sometimes to excess. The reasons for excessive inhibition vary. Some people tend to inhibit feelings because they do not trust their ability to control them. Others do so because they are confused by some emotions and prefer to avoid dealing directly with them. At times, some individuals feel awkward or even guilty about their feelings and are insecure about sharing them with others or displaying them openly. Regardless of the cause, when emotions are inhibited from expression excessively the consequence is a painful and sometimes disorganizing burden for the individual. When this becomes a trait-like feature, the resulting internalization of affect can easily create a predisposition to any of a variety of somatic difficulties such as headache, stomach or intestinal problems, blood pressure irregularities, etc., and of course, can contribute significantly to the onset of affective disruptions such as tension, anxiety and depression.. These findings are consistent with the study of Hagglof, Andren, Bergstrom, Marklund, Wendelius, (1998) and the study of

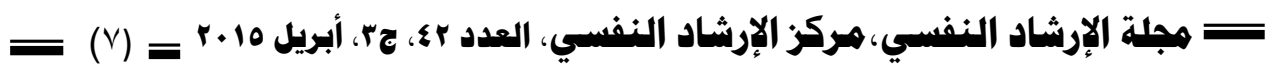




\section{Personality Attributes of Secondary Nocturnal Enuresis}

Theunis, Van Hoecke, Paesbrugge, Hoebeke, Vande Walle (2002) which found that such children experience a loss of self-esteem, although this appears to be specific to certain aspects of functioning, such as perceived social competence and physical appearance.

In disagreement with this study, Hoecke, Hoebeke, Braet and Walle (2004) found no evidence of internalizing problems (anxiety/depression) and low self-esteem in the self-report of enuretic children. In contrast, parents rate enuretic children as having more internalizing problems.

The enuretics also suffer from repressive processes as it appears from fewer blends. To create a blend response, the person must appreciate the complexity of the inkblot, which requires both analysis and synthesis. All people are complex to some extent, and some are more complex than others, but the level of complexity in any person is not static. Instead, it tends to increase or decrease around some level that is idiosyncratically typical for the specific individual. For instance, people who are very intelligent usually are more psychologically complex than those whose intellectual levels are average or below average. However, at any given time, that situation could reverse depending on the experiences of stress, unfulfilled needs, unresolved conflicts, etc. that might exist within a person. As stresses, needs, and conflicts become modest or even minimal, complexity will decrease to some extent but as the experience of stresses, needs, conflicts, etc. increases, so too does the level of complexity. Usually, there will be one or more blends in a person's protocol. A complete absence of blends suggests narrowness and constriction. This is consistent with the finding that blends are less frequent in the protocols of depressives and persons with belowaverage intelligence. In contrast, an extremely high number of blends (eight or more) suggests an unusual amount of complexity, to the extent that the person may be overly burdened.

Ego-centricity Index defined as $3 r+(2) / R$ indicates that the enuretic's self-worth is quite negative and he or she feels conflicted regarding a self-image. Such individuals regard themselves less favorably when compared to others. This may lead to mood fluctuations along with dysfunctional behaviors. Lack of sufficient self-concern. Low self evaluation is created by excessive concern for

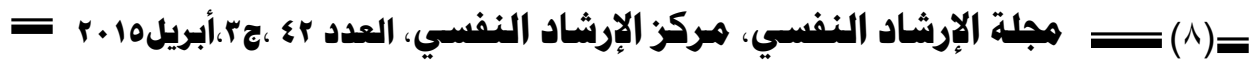


others and values of the external world. Possible self contempt. There may be some self-destructive or self-abusive behavior, but this is usually not associated with suicide risk. This characteristic is often a precursor to depression.

The study also found that enuretics have impairments in the internal representations of self and others as it appears from significantly fewer COP and fewer Pure H. They both include projected elements that are added by the person giving the answer. As self-representations, they sometimes provide useful information about internal sets that a person may have concerning interactions among people. If there is an absence of scores on either category, it suggests that the individual will be aloof, somewhat uncomfortable in social situations, and on the periphery of group situations. Given the above interpretations, it might be speculated that such deficits may complicate the internalization of experiences of coregulation with caregivers and teachers. This may leave children with a diminished capacity to evaluate their own actions and may further undermine their ability to draw on inner resources to delay gratification, inhibit behavior, and regulate emotional responses--abilities that are crucial to engaging in mutual social interactions.

\section{Conclusion:}

Children with secondary nocturnal enuresis have low selfesteem, some psychological problems such as anxiety, depression, withdrawal and impairments in the internal representations of self and others. Therefore, it is recommended to make programs for raising parent awareness regarding nocturnal enuresis and how to treat this state. Programs to enhance self-esteem and reduce anxiety and depression in the enuretic child should be in mind, as it remains unclear whether these personality attributes are a causal factor in the pathogensis of enuresis or a consequence of this disturbance.

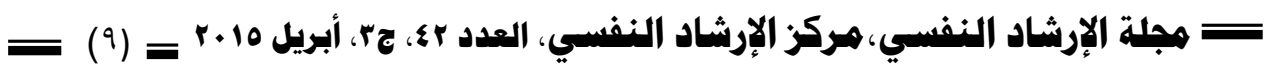




\section{References:}

1. Ames, L. B., Metraux, R. W., Walker, R. N. (1959). Adolescent Rorschach Responses Developmental Trends from Ten to Sixteen years. United States of America, Paul B. Hoeber, Inc.

2. Austin PF, Bauer SB, Bower W, Chase J, Franco I, Hoebeke P, et al. (2014). The Standardization of Terminology of Lower Urinary Tract Function in Children and Adolescents: Update Report from the Standardization Committee of the International Children's Continence Society. J Urol.

3. Coppola, G., Costantini, A., Gaita, M. \& Saraulli, D. (2011). Psychological correlates of enuresis: a case-control study on an Italian sample, Pediatr Nephrol , 26:1829-1836.

4. Exner, J. (1974). The Rorschach A Comprehensive System. Vol. (1), United States of America, John Wiley \& Sons. Inc.

5. Exner, J. (1978). The Rorschach A Comprehensive System, Current state and advanced Interpretation. Vol.(2), United States of America, John Wiley \& Sons. Inc.

6. Hagglof B, Andren O., Bergstrom E., Marklund L., Wendelius, M. (1998). Self-esteem in children with nocturnal neuresis and urinary incontinence: improvement of self-esteem after treatment. Eur Urol, 33(Supp13):16-9.

7. Hammad, E. M., El-Sedfy, G. O., Ahmed, S. M. (2005). Prevalence and risk factors of nocturnal enuresis in a rural area of Assiut governorate. Alexandria J Pediatr; 19(2):429-36.

8. Hoecke, E., Hoebeke, P., Braet, C., Walle , J. (2004). AN ASSESSMENT OF INTERNALIZING PROBLEMS IN CHILDREN WITH ENURESIS. The Journal of Urology, Volume 171, Issue 6, Part 2, , Pages 2580-2583

9. Mellon, M.W., Natchev, B. E., Katusic, S.K., Colligan, R. C., Weaver, A. L., Voigt RG, et al. (2013). Incidence of enuresis and encopresis among children with attention deficit/hyperactivity disorder in a population-based birth cohort. Acad Pediatr;13:322-7.

10. Mohammed, A. H. Saleh, A. G. Al Zoheiry, I. (2014). Frequency of bedwetting among primary school children in Benha city, Egypt. The Egyptian Journal of Medical Human Genetics, 15, 287-292

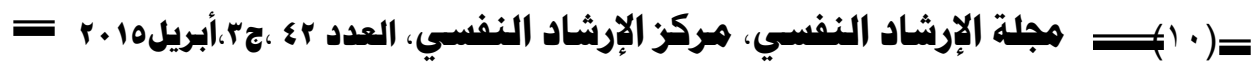


11. National Kidney Foundation Home Page. Available at: http:// www.kidney.org/patients/BW. Accessed December 4, 2003

12. Nevéus T, von Gontard A, Hoebeke P, et al. (2006). The standardization of ter- menology of lower urinary tract function in children and adolescents: report from the Standardization Committee of the International Children's Continence Society. $J$ Urol.;176:314-24.

13. Robson WL, Leung AK. (2000). Secondary nocturnal enuresis. Clin Pediatr (Phila);39:379-85.

14. Safarinejad MR. (2007). Prevalence of nocturnal enuresis, risk factors, associated familial factors and urinary pathology among school children in Iran.. J Pediatr Urol.;3:443-52.

15. Theunis M., Hoecke, V., Paesbrugge S., Hoebeke P., Vande Walle, J. (2002). Self-image and performance in children with nocturnal enuresis. Eur Urol.;41:660-667.

16. Von Gontard, A., Mauer-Mucke K, Plück J, Berner W, Lehmkuhl G. (1999). Clinical behavioral problems in day-and night-wetting children. Pediatr Nephrol;13:662-7.

17. Yu, B. Kong, F. Peng, M. Ma, H. Liu, N. Guo, Q. (2012). Assessment of memory/attention impairment in children with primary nocturnal enuresis: A vowels-based morphometry study European, Journal of Radiology, Volume 81, Issue 12, Pages 4119-4122. 